\title{
Generalized Labeled Multi-Bernoulli Extended Target Tracking Based on Gaussian Process Regression
}

\author{
Luo-jia Chi, Xin-xi Feng and Lu Miao \\ School of Information and Navigation, Air Force Engineering University, Xi'an, China
}

\begin{abstract}
For the problems that Gamma Gaussian Inverse Wishart Cardinalized Probability Hypothesis Density (GGIW-CPHD) filter cannot accurately estimate the extended target shape and has a bad tracking performance under the condition of low SNR, a new generalized labeled multi-Bernoulli algorithm based on Gaussian process regression is proposed. The algorithm adopts the star convex to model the extended target, and realizes the online learning of the Gaussian process by constructing the state space model to complete the estimation of the extended target shape. At the same time, in the low SNR environment, the target motion state is tracked by the good tracking performance of the generalized label Bernoulli filter. Simulation results show that for any target with unknown shape, the proposed algorithm can well offer its extended shape and in the low SNR environment it can greatly improve the accuracy and stability of target tracking.
\end{abstract}

\section{Introduction}

In an extended target tracking problem, target measurements collected by the sensor are no longer one point for one target, but a set of points. By obtaining the extended form of the target from measurement sets, we can achieve better tracking and recognition performance. The extended target form can be modelled as circle, rectangle, ellipse, star convex and other geometric shapes. In 2008, Koch first proposed the random matrix ${ }^{[1]}$ which modelled the target as ellipse and estimated the motion state and extended form of the target. In 2012, Granstrom applied the random matrix to the extended target probability hypothesis density(PHD) filter and proposed the Gaussian Inverse Wishart PHD (GIWPHD)filter ${ }^{[2]}$, then he applied it to the Cardinalized probability hypothesis density (CPHD) filter and got Gamma Gaussian inverse Wishart CPHD (GGIWCPHD)filter ${ }^{[3]}$. However, in low SNR environment, the tracking performance of above RFS algorithm is greatly reduced. In 2008, Baum proposed an extended target tracking method based on random hypersurfaces ${ }^{[4]}$. Then he modelled the targets as star-convex ${ }^{[5]}$ and realized the tracking of irregular shape targets. In 2015, Gaussian process regression of machine learning is proposed to estimate extended target shape ${ }^{[6]}$, avoiding complex calculation problems of the above algorithm due to excessive parameters, and achieving the accurate estimation of the target of any unknown shape.

A new generalized labeled multi-Bernoulli algorithm based on Gaussian process regression is proposed to solve the problem that GGIW-CPHD filter cannot accurately estimate the extended target shape and has a bad tracking performance under the condition of low
SNR. The algorithm improves the accuracy and stability of target tracking in low SNR environment and realizes the accurate estimation of the unknown extended target shape using the Gaussian process regression.

\section{Star convex model for extended target based on Gaussian process}

\subsection{Gaussian process regression}

At present, Gaussian process is widely used in machine learning, statistics and communication processing to classify and identify signals ${ }^{[7-9]}$. In simple terms, the Gaussian process can be regarded as a distribution function $f(u)$ and can be defined by means functions $\mu(u)$ and covariance function $k\left(u, u^{\prime}\right)$.

$$
f(u) \sim G P\left(\mu(u), k\left(u, u^{\prime}\right)\right)
$$

which

$$
\begin{gathered}
\mu(u)=E[f(u)] \\
k\left(u, u^{\prime}\right)=E\left[(f(u)-\mu(u))\left(f\left(u^{\prime}\right)-\mu\left(u^{\prime}\right)\right)^{T}\right]
\end{gathered}
$$

The Gaussian process learns the unknown function through the input training data. The measurement model is given below:

$$
z_{k}=f\left(u_{k}\right)+e_{k}
$$


The learning process mainly is: given a set of measurement $z \triangleq\left[z_{1}, \ldots, z_{N}\right]^{T}$ and their corresponding input $\boldsymbol{u} \triangleq\left[u_{1}, \ldots, u_{N}\right]^{T}$, then learning the function $f$ by Gaussian process so that we can estimate the corresponding function values $f \triangleq\left[f\left(u_{1}^{f}\right), \ldots, f\left(u_{N^{f}}^{f}\right)\right]^{T} \quad$ when the input is $\boldsymbol{u}^{f} \triangleq\left[u_{1}^{f}, \ldots, u_{N^{f}}^{f}\right]^{T}$. According to literature 6 , it is known that the measurement value $z_{k}$ and function value $f$ are subject to the joint Gaussian distribution.

$$
\left[\begin{array}{l}
z_{k} \\
\boldsymbol{f}
\end{array}\right] \sim N\left(0, \quad\left[\begin{array}{ll}
k\left(u_{k}, u_{k}\right)+R & K\left(u_{k}, \boldsymbol{u}^{f}\right) \\
K\left(\boldsymbol{u}^{f}, u_{k}\right) & K\left(\boldsymbol{u}^{f}, \boldsymbol{u}^{f}\right)
\end{array}\right]\right)
$$

From the above joint Gaussian distribution, it is easy to obtain the likelihood function and the initial prior probability:

$$
\begin{gathered}
p\left(z_{k} \mid \boldsymbol{f}\right)=N\left(z_{k} ; H_{k}^{f} \boldsymbol{f}, R_{k}^{f}\right) \\
p(\boldsymbol{f})=N\left(0 ; P_{0}^{f}\right)
\end{gathered}
$$

which

$$
\begin{gathered}
H^{f}\left(u_{k}\right)=K\left(u_{k}, \boldsymbol{u}^{f}\right)\left[K\left(\boldsymbol{u}^{f}, \boldsymbol{u}^{f}\right)\right]^{-1} \\
R^{f}\left(u_{k}\right)=k\left(u_{k}, u_{k}\right)+R-K\left(u_{k}, \boldsymbol{u}^{f}\right)\left[K\left(\boldsymbol{u}^{f}, \boldsymbol{u}^{f}\right)\right]^{-1} K\left(\boldsymbol{u}^{f}, u_{k}\right)
\end{gathered}
$$

$$
P_{0}^{f}=K\left(u^{f}, u^{f}\right)
$$

Through (6) the likelihood function is obtained, then establish the state space model, and the Gaussian process regression can be realized using the Kalman filter.

$$
\begin{gathered}
f_{k+1}=F^{f} f_{k}+Q^{f}, \quad w_{k}^{f} \sim N\left(0, Q^{f}\right) \\
z_{k}=H^{f}\left(u_{k}\right) f_{k}+e_{k}^{f}, \quad e_{k}^{f} \sim N\left(0, R^{f}\left(u_{k}\right)\right) \\
f_{0} \sim N\left(0, P_{0}^{f}\right)
\end{gathered}
$$

which

$$
\begin{gathered}
F^{f}=e^{-\alpha T} I \\
Q^{f}=\left(1-e^{-2 \alpha T}\right) K\left(u^{f}, u^{f}\right)
\end{gathered}
$$

\subsection{Star convex model for extended target based on Gaussian process}

In this paper, the extended target shape is modeled by star convex, and the estimation of it is realized by combining the learning characteristics of Gaussian process regression. According to the definition of star convex model, the shape of the target can be represented by the radius function $r=f(\theta)$. When the measurements from the sensor come from target surface, we model the measurement equation as

$$
\boldsymbol{z}_{k, l}=c_{k}+s_{k, l} \boldsymbol{p}\left(\theta_{k, l}\right) f\left(\theta_{k, l}\right)+\boldsymbol{e}_{k, l}
$$

the location of the object at time $k$ is $c_{k},\left\{z_{k, l}\right\}_{l=1}^{N_{z, k}}$ is the measurement set at time $k,\left\{\theta_{k, l}\right\}_{l=1}^{N_{z, k}}$ and $f\left(\theta_{k, l}\right)$ are respectively measured corresponding angles and radius, $\boldsymbol{p}\left(\theta_{k, l}\right)$ is the direction vector, $s_{k, l}$ is the scaling factor, $e_{k, l}$ is the Gaussian noise with zero mean and covariance $R$.

The radius function of the target shape can be learned through the Gaussian process

$$
f(\theta) \sim G P\left(\mu(\theta), k\left(\theta, \theta^{\prime}\right)\right)
$$

For each measurement value $z_{k, l}$, it corresponds to an angle $\theta_{k, l}^{L}$. Taking into account the initial direction $\psi_{k}$ of the target, the expression of $\theta_{k, l}^{L}$ is:

$$
\begin{aligned}
\theta_{k, l}^{L}\left(c_{k}, \psi_{k}\right) & =\angle\left(z_{k, l}-c_{k}\right)-\psi_{k} \\
& =\theta_{k, l}^{G}\left(c_{k}\right)-\psi_{k}
\end{aligned}
$$

Put the formula (11) and (16) in(14), the standard measurement equation can be obtained, and its expression is:

$$
\begin{aligned}
z_{k, l} & =c_{k}+s_{k, l} p_{k, l}\left(c_{k}\right) f\left(\theta_{k, l}^{L}\left(c_{k}, \psi_{k}\right)\right)+e_{k, l} \\
& =c_{k}+s_{k, l} p_{k, l}\left(c_{k}\right)\left[H^{f}\left(\theta_{k, l}^{L}\left(c_{k}, \psi_{k}\right)\right) f_{k}+e_{k, l}^{f}\right]+\bar{e}_{k, l} \\
& =\underbrace{c_{k}+s_{k, l} \tilde{H}_{l}\left(c_{k}, \psi_{k}\right) f_{k}}_{h(c, \psi, f)}+\underbrace{s_{k, l} p_{k, l}\left(c_{k}\right) e_{k, l}^{f}+\bar{e}_{k, l}}_{e} \\
& =h_{k, l}\left(c_{k}, \psi_{k}, f_{k}\right)+e_{k, l}, \quad e_{k, l} \sim N\left(0, R_{k, l}\right)
\end{aligned}
$$

which

$$
\begin{gathered}
\tilde{H}_{l}\left(c_{k}, \psi_{k}\right)=p_{k, l}\left(c_{k}\right) H^{f}\left(\theta_{k, l}^{L}\left(c_{k}, \psi_{k}\right)\right) \\
h_{k, l}\left(c_{k}, \psi_{k}, f_{k}\right)=c_{k}+\tilde{H}_{l}\left(c_{k}, \psi_{k}\right) f \\
R_{k, l}=p_{k, l} R_{k, l}^{f} p_{k, l}^{T}+R \\
p_{k, l}=p_{k, l}\left(c_{k}\right)=p\left(\theta_{k, l}^{G}\left(c_{k}\right)\right)=\frac{z_{k, l}-c_{k}}{\left\|z_{k, l}-c_{k}\right\|} \\
R_{k, l}^{f}=R^{f}\left(\theta_{k, l}^{L}\left(c_{k}, \psi_{k}\right)\right. \\
s_{k, l} \sim N\left(\mu_{s}, \sigma_{s}^{2}\right)
\end{gathered}
$$

\section{Generalized labeled multi-Bernoulli algorithm based on Gaussian process regression}

\subsection{Generalized labeled Multi-Bernoulli filter}

In multiple target tracking problem, filtering methods based on traditional RFS theory cannot provide target track, therefore, Vo introduced label into RFS and put forward the general labeled multi-Bernoulli filter $(\mathrm{GLMB})^{[10][11]}$. In the process of Bayesian recursion, the posterior probability density of GLMB RFS has the characteristics of maintaining the same form, which can avoid the complicated integral solving process in 
traditional algorithm. The posterior probability density can be expressed as follows:

$$
\begin{aligned}
& \pi(X)=\Delta(X) \sum_{(I, \xi) \in F(L) \times \Theta} \omega(I, \xi) \delta_{I}[L(X)][p(\cdot ; \xi)]^{X} \\
& X=\left\{(x, l)_{i}\right\},(i=1,2, \cdots,|X|) \text { represents the labeled }
\end{aligned}
$$
RFS; $\Delta(X)$ is the labeled indicator function; $L$ is the discrete labeled space, $F(L)$ represents the track set; $\Theta$ is the discrete association space; Each pair of $(I, \xi)$ represents an association hypothesis, $\omega(I, \xi)$ is the corresponding weight; $p(x, l ; \xi)$ is the single target probability distribution; $[p(\cdot ; \xi)]^{X}=\prod_{(x, l) \in X} p(x, l ; \xi)$.

Now assuming that the posterior probability density at time $k-1$ has the form of formula (24), then the prediction step and update step of GLMB can be expressed as follows:

Prediction step:

$$
\bar{\pi}_{+}(X)=\Delta(X) \sum_{\xi, J, L_{+}} \bar{\omega}_{+}\left(\xi, J, L_{+}\right) \delta_{J \cup L_{+}}[L(X)]\left[\bar{p}_{+}(\cdot ; \xi)\right]^{X}
$$

which

$$
\begin{gathered}
\bar{\omega}_{+}\left(\xi, J, L_{+}\right)=1_{F\left(B_{+}\right)}\left(L_{+}\right) r_{B,+}^{L_{+}}\left[1-r_{B,+}\right]^{B_{+}-L_{+}} \\
\times \sum_{I \in F(L)} 1_{F(I)}(J)\left[\bar{P}_{S}(\cdot ; \xi)\right]^{J}\left[1-\bar{P}_{S}(\cdot ; \xi)\right]^{I-J} \omega(I, \xi) \\
\bar{P}_{S}(\cdot, l ; \xi)=\left\langle p(\cdot, l ; \xi), P_{S}(\cdot, l)\right\rangle \\
\bar{p}_{+}(\cdot ; \xi)=1_{L}\left(l_{+}\right) \frac{\left\langle P_{S}\left(\cdot, l_{+}\right) f_{+}\left(x_{+} \mid \cdot, l_{+}\right), p\left(\cdot, l_{+} ; \xi\right)\right\rangle}{\bar{P}_{S}\left(\cdot, l_{+} ; \xi\right)} \\
+1_{B_{+}}\left(l_{+}\right) p_{B,+}\left(x_{+}, l_{+}\right)
\end{gathered}
$$

$\xi \in \Theta, J \in F(L), L_{+} \in F\left(B_{+}\right), B_{+}$is the new target labeled space and $L \cap B_{+}=\varnothing ; r_{B,+}$ and $p_{B,+}\left(x_{+}, l_{+}\right)$are the birth probability and probability distribution of newborn target respectively; $f_{+}\left(x_{+} \mid \cdot, l_{+}\right)$is the Markov state transition function and the label of the survival target remains unchanged in prediction step. $P_{S}\left(\cdot, l_{+}\right)$is the survival probability for the target.

Update step:

$$
\pi_{Z_{+}}(X) \propto \Delta(X) \sum_{\xi, J, L_{+}, \theta_{+}} \omega_{Z_{+}}\left(\xi, J, L_{+}, \theta_{+}\right) \delta_{J \cup L_{+}}[L(X)]\left[p_{Z_{+}}\left(\cdot ; \xi, \theta_{+}\right)\right]^{X}
$$

which

$$
\begin{gathered}
\omega_{Z_{+}}\left(\xi, J, L_{+}, \theta_{+}\right)=1_{\Theta_{+}\left(J \cup L_{+}\right)}\left(\theta_{+}\right)\left[\bar{\psi}_{Z_{+}}\left(\cdot ; \xi, \theta_{+}\right)\right]^{J \cup L_{+}} \bar{\omega}_{+}\left(\xi, J, L_{+}\right) \\
\bar{\psi}_{Z_{+}}\left(\cdot, l_{+} ; \xi, \theta_{+}\right)=\left\langle\bar{p}_{+}\left(\cdot, l_{+} ; \xi\right), \psi_{Z_{+}}\left(\cdot, l_{+} ; \theta_{+}\left(l_{+}\right)\right)\right\rangle \\
p_{Z_{+}}\left(\cdot ; \xi, \theta_{+}\right)=\frac{\bar{p}_{+}(\cdot ; \xi) \psi_{Z_{+}}\left(\cdot ; \theta_{+}\left(l_{+}\right)\right)}{\bar{\psi}_{Z_{+}}\left(\cdot, l_{+} ; \xi, \theta_{+}\right)} \\
\psi_{Z_{+}}\left(\cdot ; \theta_{+}\left(l_{+}\right)\right)=\left\{\begin{array}{l}
\frac{P_{D}\left(x_{+}, l_{+}\right) g\left(z_{\theta_{+}\left(l_{+}\right)} \mid x_{+}, l_{+}\right)}{\kappa\left(z_{\theta_{+}\left(l_{+}\right)}\right)}, \theta_{+}\left(l_{+}\right)>0 \\
1-P_{D}\left(x_{+}, l_{+}\right), \theta_{+}\left(l_{+}\right)=0
\end{array}\right.
\end{gathered}
$$

$\theta_{+} \in \Theta_{+}$represents the correlation mapping of targets to measurements; $g\left(z_{\theta_{+}\left(l_{+}\right)} \mid x_{+}, l_{+}\right)$is the measurement likelihood function; $P_{D}\left(x_{+}, l_{+}\right)$is the target detection probability.

\subsection{Generalized labeled multi-Bernoulli algorithm based on Gaussian process regression}

According to the derivation of section 2.2, the extended target is modeled as

$$
\begin{gathered}
x_{k+1}=F x_{k}+w_{k}, \quad w_{k} \sim N(0, Q) \\
z_{k}=h_{k}\left(x_{k}\right)+e_{k}, \quad e_{k} \sim N\left(0, R_{k}\right) \\
x_{0} \sim N\left(\mu_{0}, P_{0}\right)
\end{gathered}
$$

The state vector of the target is expressed as $x_{k} \triangleq[\underbrace{\left(c_{k}\right)^{T},\left(c_{k}^{\prime}\right)^{T}, \psi_{k}}_{x_{k}^{n}},\left(f_{k}\right)^{T}]^{T}$, including the target's motion state $x_{k}^{n}=\left\{\left(c_{k}\right)^{T},\left(c_{k}^{\prime}\right)^{T}, \psi_{k}\right\}$ and the target expansion state $f_{k}$. The extended target measurements of each moment is $z_{k}=\left[\begin{array}{lll}z_{k, 1}^{T}, \ldots, & z_{k, n}^{T}\end{array}\right]^{T}$.

which

$$
\begin{aligned}
& R_{k}=\operatorname{diag}\left[R_{k, 1}, \ldots, R_{k, n}\right] \\
& h_{k}\left(x_{k}\right)=\left[h_{k, 1}\left(x_{k}\right)^{T}, \ldots, h_{k, n}\left(x_{k}\right)^{T}\right]^{T} \\
& F=\operatorname{diag}\left(F^{n}, F^{f}\right) \\
& Q=\operatorname{diag}\left(Q^{n}, Q^{f}\right)
\end{aligned}
$$

$F^{f} 、 Q^{f}$ are determined by formula (12) and (13) which related to the movement of the target. The specific parameters are given in the simulation experiment.

The main steps for the generalized labeled multiBernoulli algorithm based on Gaussian process regression are that firstly using generalized labeled multi-Bernoulli filter to obtain the target motion state, then according to formula (17) derived in this paper, Gaussian process regression is realized by using kalman filter to complete the estimate of the target extended shape. The joint posterior probability density is

$$
p\left(f_{k}, x_{0: k}^{n} \mid z_{1: k}\right) \approx p\left(f_{k} \mid x_{0: k}^{n}, z_{1: k}\right) p\left(x_{0: k}^{n} \mid z_{1: k}\right)
$$

$p\left(f_{k} \mid x_{0: k}^{n}, z_{1: k}\right)$ is obtained by Gaussian process learning, $p\left(x_{0: k}^{n} \mid z_{1: k}\right)$ is given by generalized labeled multi-Bernoulli filter.

\section{Simulation result and analysis}

\subsection{Parameter setting}

This paper makes a comparative analysis of the proposed algorithm and GGIW-CPHD algorithm. we verify the performance of the proposed algorithm in the two following aspects:

(1) shape estimation: whether the target of any unknown shape can be well studied and given its extended form; 
(2) tracking performance: whether it has good tracking accuracy and stability under low SNR environment.

Our experiment assumes that the extended target is shaped like a star. The new target random set can be represented by the labeled Bernoulli parameter set $\left\{r_{B, k}\left(l_{i}\right), p_{B, k}\left(l_{i}\right)\right\}_{i=1}^{4}$. The probability density of new target is $r_{B, k}\left(l_{i}\right)=0.04$ which is subject to Gaussian distribution $p_{B}\left(l_{i}\right)=N\left(x ; m_{B}^{(i)}, P_{B}\right)$. The initial state of the four new targets is $m_{B}^{(1)}=[40,2.5,40,2.5]^{\mathrm{T}}$, $m_{B}^{(2)}=[18,-2.5,20,1.5]^{\mathrm{T}} \quad, \quad m_{B}^{(3)}=[30,3,130,0]^{\mathrm{T}} \quad$, $m_{B}^{(4)}=[120,2,190,-3]^{\mathrm{T}} . P_{B}=\operatorname{diag}\left([10,5,10,5]^{\mathrm{T}}\right)^{2} . \quad$ Target survival probability $P_{s}=0.98$, detection probability $P_{D}=0.8$. The background clutter number obeys the Poisson distribution with a mean value of 20 and evenly distributed in the monitoring area. The maximum Gaussian number $H_{\max }=1000$. Target position and rotation direction are modeled as the uniform velocity model:

$$
F^{n}=\left[\begin{array}{ll}
1 & \mathrm{~T} \\
0 & 1
\end{array}\right] \otimes I_{3}, Q^{n}=\left[\begin{array}{cc}
\frac{T}{3} & \frac{T}{2} \\
\frac{T}{2} & T
\end{array}\right] \otimes\left[\begin{array}{ccc}
\sigma_{q}^{2} & 0 & 0 \\
0 & \sigma_{q}^{2} & 0 \\
0 & 0 & \sigma_{q^{\prime}}^{2}
\end{array}\right]
$$

Assuming the position process noise $\sigma_{q}^{2}=1$, rotation direction process noise $\sigma_{q^{\prime \prime}}^{2}=0.1$, sampling period $T=1$. The covariance function of Gaussian process ${ }^{[6]}$ is

$$
k\left(\theta, \theta^{\prime}\right)=\sigma_{f}^{2} e^{-\frac{2 \sin ^{2}\left(\frac{\theta-\theta^{\prime}}{2}\right)}{l^{2}}}+\sigma_{r}^{2}
$$

The radius prior variance $\sigma_{r}^{2}=1$, output prior variance amplitude $\sigma_{f}^{2}=2$, length scale of the function $l=\frac{\pi}{4}$ and the measurement noise covariance $R=0.1^{2} I_{2}$. The whole tracking process continuous 100 s and 100 Monte Carlo experiment is conducted. The initial state of the target is shown in table 1 .

\begin{tabular}{|c|c|c|c|}
\hline \multirow[b]{2}{*}{ Target } & \multicolumn{3}{|c|}{ State } \\
\hline & Initial state & $\begin{array}{c}\text { Appearing } \\
\text { time }\end{array}$ & $\begin{array}{c}\text { Disappearing } \\
\text { time }\end{array}$ \\
\hline Target 1 & {$\left[\begin{array}{lllll}40 & 2.5 & 40 & 2.5\end{array}\right]$} & $1 \mathrm{~s}$ & $60 \mathrm{~s}$ \\
\hline Target 2 & {$\left[\begin{array}{llll}180 & -2.5 & 20 & 1.5\end{array}\right]$} & $21 \mathrm{~s}$ & $80 \mathrm{~s}$ \\
\hline Target 3 & {$\left[\begin{array}{lllll}30 & 3 & 130 & 0\end{array}\right]$} & $31 \mathrm{~s}$ & $100 \mathrm{~s}$ \\
\hline Target 4 & {$\left[\begin{array}{llll}120 & 2 & 190 & -3\end{array}\right]$} & $41 \mathrm{~s}$ & $100 \mathrm{~s}$ \\
\hline
\end{tabular}

Table 1 Target initial state

\subsection{Analysis of simulation result}

In this paper, the simulation results are shown in figure 1-4. Fig.1 shows the extended target real tracks and filtering results of GGIW-CPHD and our proposed algorithm.

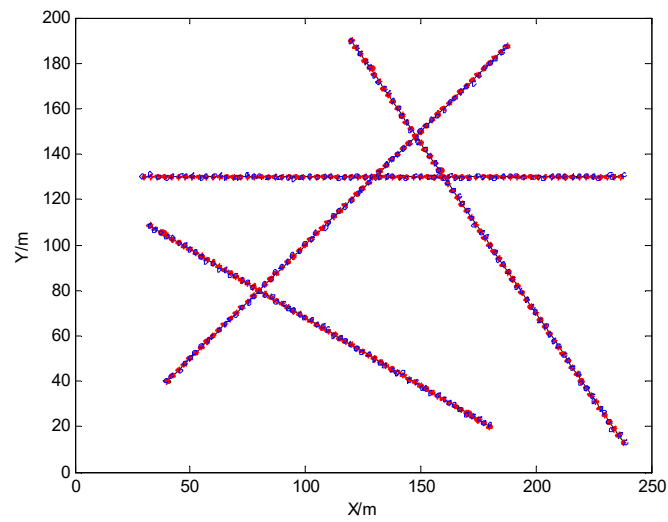

Fig. 1. Target real trails and filtering results.

Fig. 2 shows the local amplification of filtering results. The black line, blue line and red line are respectively stand for target real tracks and real shape of the extension、GGIW-CPHD filtering results and shape estimation 、 our proposed algorithm filtering results and shape estimation. We can see intuitively from Fig.1、2 that in a low SNR environment, our proposed algorithm can effectively suppress clutter measurement interference and get better and sustained tracking results. Meanwhile, the proposed algorithm is more precise in shape estimation than GGIW-CPHD.

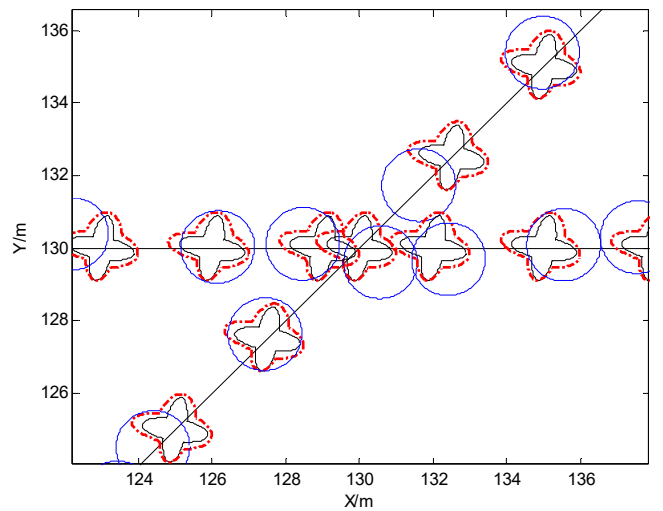

Fig. 2. Local amplification of filtering results.

Fig.3 shows target estimation number of GGIWCPHD and the proposed algorithm. Yellow, green shadows respectively stand for target number statistical standard deviation of GGIW - CPHD and the proposed algorithm. We can see that GGIW - CPHD and the proposed algorithm both carry on the unbiased estimation, but the standard deviation of GGIW-CPHD is bigger than the proposed algorithm which verifies that in low SNR environment, our proposed algorithm has better tracking accuracy and stability. 


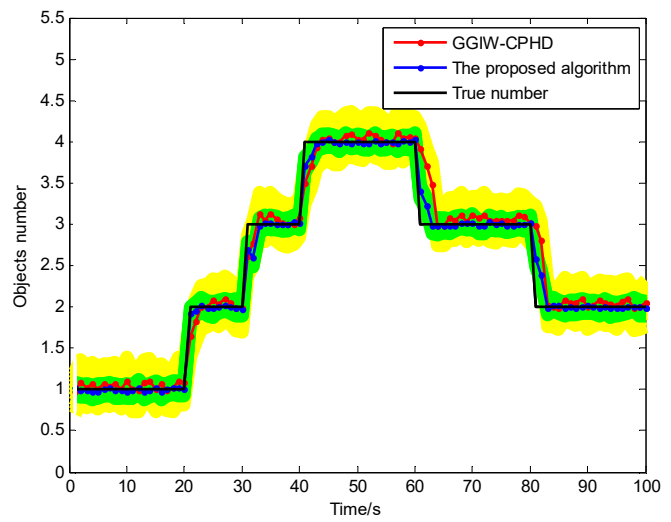

Fig. 3. Estimation of target number.

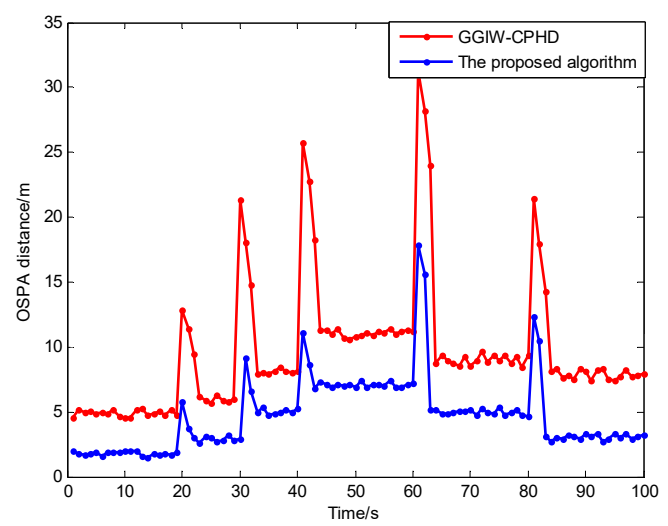

Fig. 4. OSPA distance contrast.

Fig.4 shows OSPA distance contrast of GGIWCPHD and the proposed algorithm. It can be seen from the figure, when target number changes, the filtering effect of both algorithm has varying degrees of decline, but the proposed algorithm is smaller on the whole and proves to have a higher filtering accuracy and stability.

\section{Conclusions}

This paper presents a generalized labeled Bernoulli algorithm under Gaussian process regression. The proposed algorithm uses Gaussian process of machine learning to learn extended target shape, avoiding the existing algorithms' excessive parameters calculation complex problems, then tracking the targets based on the generalized labeled Bernoulli filter good tracking performance under low SNR environment. The experimental results show that the proposed improved algorithm can effectively track the extended target in the low SNR environment, which also has better stability and more accurate shape estimation.

\section{References}

1. J .W .Koch. Bayesian approach to extended object and cluster tracking using random matrices[J]. IEEE Transactions on Aerospace and Electronic Systems, 2008, 44(3): 1042-1059.
2. K. Granstrom, U. Orguner. A PHD Filter for Tracking Multiple Extended Targets Using Random Matrices[J]. IEEE Transactions on Signal Processing, 2012, 60(11): 5657-5671.

3. C. Lundquist, K. Granström, U. Orguner. An Extended Target CPHD Filter and a Gamma Gaussian Inverse Wishart Implementation[J]. Journal of Selected Topics in Signal Processing, 2013, 7(3): 472-483.

4. M. Baum, U. D. Hanebeck. Random Hypersurface Models for Extended Object Tracking[C]. IEEE International Symposium on Signal Processing and Information Technology. IEEE, 2010: 178-183.

5. M. Baum, U. D. Hanebeck. Shape tracking of extended objects and group targets with star-convex RHMs[C].Proceedings of the, International Conference on Information Fusion. IEEE, 2011: 1-8.

6. N. Wahlström, E. Özkan. Extended Target Tracking Using Gaussian Processes[J]. IEEE Transactions on Signal Processing, 2015, 63(16): 4165-4178.

7. C. E. Rasmussen, C. K. I. Williams. Gaussian Processes for Machine Learning [M]. Cambridge, USA :The MIT Press, 2005.

8. J. Ko, D. Fox. GP-BayesFilters: Bayesian Filtering Using Gaussian Process Prediction and Observation Models[J]. Autonomous Robots, 2009, 27(1): 75-90.

9. E. Özkan, N. Wahlström, S. J. Godsill. RaoBlackwellised particle filter for star-convex extended target tracking models[C]. International Conference on Information Fusion. IEEE, 2016.

10. B. T. Vo, B. N. Vo. Labeled random finite sets and multi-object conjugate priors[J]. IEEE Transactions on Signal Processing, 2013, 61 (13): 3460-3475.

11. B. N. Vo, B. T. Vo, Phung D. Labeled random finite sets and the Bayes multi-target tracking filter[J]. IEEE Transactions on Signal Processing, 2014, 62 (24): 6554-6567. 